\title{
The Application of the Complementary and Alternative Therapies in Relieving Insomnia for Cancer Patients
}

\author{
Juan LIAO ${ }^{1}$, Bu-Xin $\mathrm{HAN}^{2, *}$, Ling TANG ${ }^{3, *}$, Dong $\mathrm{CAO}^{4}, \mathrm{Nan}_{\mathrm{ZHAO}}{ }^{1}$ \\ ${ }^{1}$ Department of Oncology, Xi Yuan Hospital, China Academy of Chinese Medical Sciences, Beijing 100091, China; \\ ${ }^{2}$ Institute of Psychology, Chinese Academy of Sciences, Beijing 100012, China \\ ${ }^{3}$ Nursing Department, The Third Affiliated Hospital of Beijing University of Chinese Medicine, Beijing 100029, China; \\ ${ }^{4}$ The Laboratory Animal Research Center, Peking University, Beijing 100871, China
}

\begin{abstract}
Objective: To observe the curative effect of the complementary and alternative therapies in relieving insomnia for cancer patients.

Method: According to the principle of randomization, with meeting the inclusion criteria, the 60 patients who were diagnosed as insomnia caused by liver qi stagnation were randomly divided into the control group and the intervention group. The intervention group (30 individuals treated with mini-needle therapy combined with TCM five elements music), the control group (30 individuals who were treated with single mini-needle therapy), the body buried needle 24 hours/time, 5 times for a course of treatment, interval of 2 days between 2 courses, a total of 8 needles embedded. Pittsburgh sleep quality index (PSQI) scores of 2 groups were analyzed using SPSS19.0 statistical software.
\end{abstract}

Results: The total effective rate was $86.1 \%$ in the intervention group, and $75.9 \%$ in the control group, the difference was statistically significant between 2 groups $(P<0.05)$. The comparison of the PSQI score between the 2 groups propost treatment showed that there were statistically significant differences $(P<0.05)$. The reduction rate of PSQI in the intervention group was $31.7 \%$, indicating that $31.7 \%$ was effective, while $23.1 \%$ in the control group was ineffective. Conclusion: The mini-needle therapy combined with TCM five elements music could relieve insomnia caused by qi stagnation of liver in patients with advanced cancer.

Key words: TCM five elements music; Mini-acupuncture; Insomnia; Qi stagnation of liver; Advanced cancer

\section{Introduction}

Malignant tumors have become relatively frequent and

\section{"Corresponding Author:}

Bu-Xin HAN, Professor, Ph. D supervisor, Boffin, Institute of Psychology, Chinese Academy of Sciences, Beijing 100012, China E-mail: hanbx@psych.cn; Ling TANG, Chief superintendent nurse, head of Nursing Department of the Third Affiliated Hospital of Beijing University of Chinese Medicine, Beijing 100029, China E-mail: zydsyhlb@126.com

Received: March 31 2019, Revised:April I5 2019, Accepted:April 20 2019, Online: May 152019 common diseases ${ }^{[1]}$. And Insomnia has become one of the serious problems for such patients undergoing chemotherapy, accounting for $54.72 \%$ of the total incidence of insomnia, which often has an impact on patients' mental health ${ }^{[2]}$, with an incidence of higher than $70 \%{ }^{[3]}$. Insomnia is a common clinical disease, 
seriously affecting the rehabilitation of patients, for which an adequate sleep is necessary to help rehabilitation ${ }^{[4]}$. Music therapy as an important part of psychological interventions ${ }^{[5]}$, can ensure patients' sleep quality ${ }^{[6-7]}$. Minineedle therapyis the development of acupuncture needles (it can be extended to the whole role of acupuncture), which is used to improve clinical efficacy, andis widely used in clinical procedures ${ }^{[8]}$. Traditional Chinese medicine (TCM) believes that insomnia is classified with in the "disorders" category, for which there are several treatment methods. In this study, 60 cases of advanced cancer patients were treated with mini-needle therapy to improve the patient's insomnia symptoms, and a half of the patients (the intervention group) were treated with addition of TCM five elements music. The results showed that the intervention group achieved beneficial effects.

\section{Materials and Methods}

\section{Ethic committee}

This study was approved by ethic committee of Xiyuan Hospital, China Academy of Chinese Medical Sciences.

\section{Inclusion criteria}

(1) Patients with stable vital signs and clear consciousness;

(2) Patients were diagnosed with malignant tumors; (3) Patients had insomnia for more than 4 weeks, and did not take psychiatric drugs or had stopped taking more than 2 weeks; (4) Before treatment, patients could accept this fact, were willing to participate in this test, and signed the informed consents; (5) Patients were not accompanied by other serious physical illness; (6) Patients had no mental disorders, and could independently complete the questionnaire survey before and after chemotherapy. (7)Diagnostic criteria of insomnia in line with Chinese Psychiatric Classification and Diagnostic Criteria (3rd edition) (CCDM-3) ${ }^{[9]}$ in the diagnosis of insomnia, and liver insomnia in line with the diagnostic criteria of Internal Medicine of Traditional Chinese Medicine ${ }^{[10]}$.

\section{Exclusion criteria}

Patients with any one of the following ware excluded: (1) Other causes or other diseases caused insomnia; (2) Patients with primary diseases of heart, liver, kidney, brain and other organs; (3) Patients with disturbance of consciousness, language communication disorders and poor degree of poor patients; (4) Patients with mental drug abuse or dependence; (5) Patients had nearly 1-week history of fever and infection. (6) Patients couldn't cooperate with the treatment.

\section{Patients}

Totally 60 cases of advanced cancer patients admitted to the oncology department of Xi Yuan Hospital, China academy of Chinese Medical Sciences from July 2016 to August 2017 were selected and diagnosed as hepatic qi stagnation type insomnia. All patients had definite history of insomnia. And because of voluntary withdrawal of 1 patient, and termination of aggravation of 1 patient, 58 cases were included ultimately, including 23 male and 35 female. According to the international TNM staging standard (UICC), there were 1 case of stage II, 1 case of stage III, 1 case of stage IIIB, and 55 cases of stage IV. The diagnosis of cancer included 12 cases of breast cancer, 14 cases of lung cancer, 9 cases of rectal cancer, 5 cases of colon cancer, 1 case of pancreatic cancer, 2 cases of ovarian cancer, 2 cases of gastric cancer and 13 cases of other diseases.

The SAS software generated random number. According to the patient into the order of random access, and distribution ratio $(1: 1)$, the 58 patients were randomly divided into control group $(n=29)$ and intervention group $(n=29)$. The intervention group received intradermal buried needle (mini-needle acupuncture) combined with TCM five elements music, and the control group received intradermal buried needle alone. The intervention group had 12 males and 17 females, aged 36-70 years old, while the control group had 11 males and 18 females, aged 36-71 years old. There was no statistically significant difference in the distribution of baseline data such as age, gender, and tumor stage between the 2 groups $(P>0.05)$.

\section{Treatment}

Control group: $0.20 \mathrm{~mm} \times 1.5 \mathrm{~mm}$ one-time sterilization of SEIRIN Co., Ltd. The selected acupoints were Xinshu, Shenshu, Ganshu, Shenmen, Neiguan, Zusanli, Sanyinjiao, Baihui, Yintang, Shendao, Zhaohai, Taichong, 
and Yongquan under the guidance of the syndrome differentiation.After the sterile disposable mini-needle is inserted into the selected acupoint, press the needle and paste it well. The mini-needle has to be buried for 48 hours; Acupoints should be pressed 4 times a day for 1 minute each time, with an interval of 4 hours. Pressure intensity is based on the patient's to lerance.

Intervention group: On the basis of single minineedle therapy, TCM five elements music therapy was supplemented for the treatment of liver qi stagnation. And melodic music with Yu tone (one of five-tone scale in TCM) and Jiao-tone (one of five-tone scale in TCM) were used, 30 minutes/time, 15 minutes for each style of music, 5 times for each course of treatment, 4 courses in total. The interval of 2 days was appropriate between 2 courses. All of the above operations are carried out after standardized training.

\section{Observational indicators}

Pittsburgh Sleep Quality Index (PSQI) table was used to assess the sleep quality of patients of 2 groups before and after treatment ${ }^{[6]}$. The table consists of 18 entries composed of 7 factors, each factor scoresranging from 0 to 3 points. The total score is 0 to 21 points. The higher the scores were, the worse the sleep quality was. There was no assessment of insomnia drugs in the PSQI scale because the intervention group did not use sedative drugs. Before treatment, the patients were observed and recorded. After 4 courses of treatment, they were observed and recorded again.

The standard of efficacy with reference toclinical efficacy criteria of Chinese Medicine New Drug Clinical Research Guidelines promulgated by the Ministry of Health in $1993^{[7]}$. Reduction rate of PSQI score $=[($ Score before treatment - Score after treatment)/Pre-treatment score] $\times 100 \%$, including (1) recovery: PSQI reduction rate up to $76 \%-100 \%$, (2) markedly: PSQI reduction rate up to $51 \%$ $75 \%$; (3) effective: PSQI reduction rate up to $25 \%-50 \%$, and (4) invalid: PSQI reduction rate $<25 \%$.

\section{Statistical analysis}

SPSS 21.0 statistical software to analyze the data, all data are used bilateral test, the enumeration data was described by percentages $(\%)$, the measurement data was described by the mean and standard deviation $(\bar{x} \pm s)$. The comparison of repeated measurement data within the group was conducted using repeated measurement ANOVA, and the comparison between groups using $t$ test, variance Analysis or rank sum test. $P<0.05$ was considered statistically significant.

\section{Results}

\section{The comparison of efficacy between both groups}

the total effective rate was $86.1 \%$ in the intervention group, and $75.9 \%$ in the control group. The efficacy of the 2 groups was compared using the rank of test, the difference was statistically significant $(P<0.05)$, suggesting that theintervention group was better than the control group. (Table 1)

\section{The comparison of the reduction rate of PSQI score between the 2 groups}

The comparison of the PSQI score between the 2 groups pro-post treatment showed that there were statistically significant differences $(P<0.05)$. The reduction rate of PSQI score in the intervention group was $31.7 \%$, indicating that $31.7 \%$ was effective, while $23.1 \%$ in the control group was ineffective. (Table 2)

Table 1 The comparison of efficacy between both groups

\begin{tabular}{lcccccc}
\hline Groups & Cases & No effect & Effect observed & $\begin{array}{c}\text { Remarkable } \\
\text { effect }\end{array}$ & Full recovery & Total effective rate (\%) \\
\hline Intervention group & 29 & $4(13.8 \%)$ & $7(24.1 \%)$ & $17(58.6 \%)$ & $1(3.4 \%)$ & 86.1 \\
Control group & 29 & $7(24.1 \%)$ & $10(34.5 \%)$ & $12(41.4 \%)$ & 0 & 75.9 \\
\hline
\end{tabular}


Table 2 The comparison of the reduction rate of PSQI score between the 2 groups $(\bar{x} \pm s)$

\begin{tabular}{|c|c|c|c|c|c|}
\hline Groups & Case & Treatment & $\begin{array}{l}\text { Total PSQI } \\
\text { (points) }\end{array}$ & $\begin{array}{c}\text { Reduction of PSQI score } \\
\text { (points) }\end{array}$ & $\begin{array}{c}\text { Reduction rate } \\
(\%)\end{array}$ \\
\hline \multirow{3}{*}{ Intervention group } & \multirow{3}{*}{29} & Pro-T & $18.36 \pm 2.41$ & \multirow{3}{*}{$5.82 \pm 1.62$} & \multirow{3}{*}{31.7} \\
\hline & & & & & \\
\hline & & Post-T & $12.54 \pm 3.54$ & & \\
\hline \multirow{3}{*}{ Control group } & \multirow{3}{*}{29} & Pro-T & $17.97 \pm 2.67$ & \multirow{3}{*}{$4.14 \pm 1.04$} & \multirow{3}{*}{23.1} \\
\hline & & & & & \\
\hline & & Post-T & $13.82 \pm 1.97$ & & \\
\hline
\end{tabular}

Notes: Pro-T refers to pro-treatment; Post-T refers to post-treatment

Table 3 The comparison of the total PSQI score pro-post treatment within each group $(\bar{x} \pm s)$ points

\begin{tabular}{|c|c|c|c|c|c|}
\hline Groups & Baseline & 2 weeks after treatment & 4 weeks after treatment & $\boldsymbol{F}$ & $P$ \\
\hline Intervention group & $18.36 \pm 2.41$ & $13.67 \pm 2.41$ & $12.54 \pm 2.41^{* \#}$ & 23.143 & 0.027 \\
\hline Control group & $17.97 \pm 2.67$ & $14.11 \pm 2.67$ & $13.82 \pm 2.67^{*}$ & 21.684 & 0.051 \\
\hline$F$ & 1.211 & 6.737 & 19.237 & & \\
\hline$P$ & 0.722 & 0.083 & 0.001 & & \\
\hline
\end{tabular}

${ }^{* \#} P<0.05$

\section{Comparison of the total PSQI score pro-post treatment within each group}

The repeated measurement ANOVA was used for the intra-group measurement. It was shown that there were statistically significant differences pro-post treatment within each group $(P<0.05)$. At the same time, there was significantly differences of the total PSQI score 4 weeks after treatment when comparing the 2 groups $(P<0.05)$. (Table 3$)$

\section{Discussion}

Insomnia is also known as wakefulness, which is mainly due to various causes of the body caused by imbalance between yin and yang. According to TCM syndrome differentiation, insomnia can be divided into 5 types, including qi stagnation of liver type, yin deficiency with fire hyperactivity type, heart and spleen deficiency type, qi deficiency of heart and gallbldadder type, and phlegmheat obstruction in the lung. The focus of this study is concerned with insomnia. Patients mainly manifested as difficult to sleep, irritability, bitter and dry mouth, etc., the pathogenesis of emotional disorders lead to liver catharsis, stagnant fire damaging the spirit. In addition to insomnia patients, also, insomnia leads to a series of clinical symptoms such as chest distress, headache, constipation, urine and other clinical symptoms. Long-term insomnia makes patients miserable, which further increases the psychological problems.

With the comprehensive advancement of China's health care reform, and the emphasis on TCM inheritance and development, the use of needle in the clinical research has been gradually deepened, and has unique advantages in improving the subjective symptoms. As a new type of modified needle, it is easy to operate, and the human body does not produce side effects similar to drugs when medical staff press the needle. As an effective TCM technology, it has been widely used in clinical practice $^{[11-14]}$. Gao et al.'s study ${ }^{[15]}$ shows that auricular acupressure has its unique advantages in improving sleep disorders and patient's daytime functional status adjustment. Li et al. ${ }^{[12]}$ reported a clinical study of the use of mini-needle therapy with rehabilitation guidelines for the treatment of knee osteoarthritis, to verify clinical efficacy of the treatment of knee osteoarthritis, and the results showed that it can be a good relief of knee pain, and a good method to improve the knee function; Shi et al. ${ }^{[16]}$ summed up the clinical study of the role for 
acupuncture therapy in pain syndrome, once again verified that acupuncture could promote qi and blood circulation, and pressing the needle and continued needle-embedding in the skin or subcutaneously could give a specific acupoints to long-lasting and gently benign stimulation, thereby enhancing the efficacy of acupuncture analgesia, and playing to prevent the recurrence of pain. As part of the psychological intervention, Chinese medicine has played an important complementary role in clinical treatment, and several studies ${ }^{[6,17-18]}$ have confirmed that music therapy can improve sleep quality. Besides having a direct impact on mood states, music therapy can have physiological effects. The mechanism is based on the auditory system and the role of the cerebral cortex, which can stimulate the body to promote physical and mental health ${ }^{[19]}$. Studies have confirmed that music therapy can improve sleep quality, increase parasympathetic activity, and regulate muscle tension and blood flow ${ }^{[20]}$. According to the theory of the TCM five elements, for hepatic qi stagnation type insomnia, the Jiao-tone music (one of five-tone scale in TCM, the equivalent of "3-mi", the third note in a musical scale according to the sol-fa system), is selected as the main tones, corresponding to wood (one of the five elements), and into the liver and gallbladder, with the functions of dispersing stagnated liver qi to relieve depression, tonifying the heart and spleen, clearing liver-fire, and soothing the nerves. At the same time, according to the principle of "Mother-supplementing", Yu-tone music (one of five-tone scale in TCM) is chosen to strengthen the kidney function, nourish the kidney yin, liver and heart, and purge theliver fire. The Yu-style music (the equivalent of 6-la) was as the main tones, corresponding to water (one of the five elements), into the kidney.

Western medicine for the intervention of insomnia is to allow patients to take anti-anxiety agents and sedative hypnotic drugs to achieve the goal of alleviating insomnia, but most of the drugs will have a normal impact on the body's normal sleep structure, resulting in clinical symptoms of patients' attention unable to focus during the day, while anti-anxiety agents and sedative hypnotic drugs addiction and withdrawal reactions severely limit the clinical use of these drugs ${ }^{[9]}$.
In this study, It was shown that there were statistically significant differences pro-post treatment within each group $(P<0.05)$. At the same time, there was significantly differences of the total PSQI score 4 weeks after treatment when comparing the 2 groups $(P<0.05)$. In this study, weused the mini-needles to effectively stimulate human body acupuncture points to nourish nerve, promote the blood circulation, for pressing the needle buried in the skin continuously and fixedly can give specific acupoints with durable, soft benign stimulation to treat disease ${ }^{[21-22]}$.

At the same time, in this study, patientsin the intervention group used to listen to TCM music, music therapists through the principle of music therapy," induced", "suggested", "supported", and "showed empathy", and used other ways to lead patients to produce a variety of psychological experience. when listening to five-element music, the sweet music can stimulate the ear canals, through which the sensation is then deliver to the special area in human brain based on the physiological resonance, to achieve the purpose of improving insomnia.

In summary, the skin buried needle technology combined with the TCM five elements music can improve the quality of life of patients with advanced liver cancer who suffer from liver qi stagnation. Additionally, this practice does not cause dependency, and it is an economical and practical method to relieve mood states.

\section{Declaration}

The authors of this article declare that they have no conflict of interest.

\section{References}

1 Song Y, Wang L, Pittas AG, et al. Blood 25-Hydroxy vitamin D levels and incident Type 2 Diabetes A meta-analysis of prospective studies. DCP, 2013, 36(5): 1422-1428.

2 Wang YN. Clinical efficacy and standardized operation of TIP sleep regulation technology in the treatment of primary insomnia. Beijing: Chinese Academy of Traditional Chinese Medicine, 2017. 
3 Xu YX, Yin XM. The effectiveness of Comprehensive treatment of nursing inimproving the quality of life in patients with advanced cancer. Qilu Nurs J, 2012, 18(27): 12-14.

4 Su XM. Effects of psychological intervention on the quality of life of elderly patients with pulmonary tuberculosis. Nation J Pract Nurs, 2012, 28(27): 7778.

5 Wang XS. Effects of nostalgic-cognitive behavioral intervention on depressive symptoms and cognitive function in the elderly. Chang Sha: Nan Hua University, 2014.

$6 \mathrm{Zu} \mathrm{BQ}$, Li XM. Meta analysis of the effect of music therapy on sleep quality. J Nurs, 2013, 28(11): 80-83.

7 Liao J, Zhao YC, Li J, et al. The effectiveness of nondrug techniques of Traditional Chinese Medicine in relieving depression \& anxiety--A randomized controlled trial. World Sci Tech-Modern Trad Chin Med, 2016, 18(8): 1386-1392.

8 Shi HF, Luo GQ, Li L. The clinical research progress of acupuncture therapy for pain syndrome. Hunan J Trad Chin Med, 2013, 29(5): 148-149.

9 Chinese Psychiatric Association. Chinese Classification and Diagnostic Criteria for Mental Disorders (ccmd-3). 3rd Edition. Jinan: Shandong Science and Technology Press, 2001.

10 WangYY. Internal Medicine of Traditional Chinese Medicine. Beijing: People's Medical Press House, 2011.

$11 \mathrm{Xu} \mathrm{W}$, Sun D, Zhang DT, et al. The effects of thumbtack needle with rehabilitation guidance in relieving arthritis in knee. Shanghai J Acupunct Moxibust, 2016, 35(8): 997-1000.

12 Li Y, Zhao MF. The clinical observation on the effects of acupuncture in relieving arthritis in knee. Modern J Intergr Trad ChinWestern med, 2011, (22): 27542755.
13 Shu SJ, Shen TL, Zhang W. The clinical observation on the effects of acupuncture in treating arthritis pain in hand and knee.Liaoning J Trad Chin Med, 2014, (3): 539-541.

14 Shen J, Xu W, Zhang DT, et al. The effects of thumbtack needle in relieving cervical vertigo of 57 cases. J Zhejiang Univ Traditional Chin Med, 2014, 38(5): 634-636.

15 Gao SF, Luo YJ. Clinical observation of auricular acupoint with mini-acupuncture intervention for primary insomnia. Guid J Trad Chin Med, 2016, 22(2): 94-95.

16 Shi HF, Luo GQ, Li L. The progress in clinical research on Thumbtack needle in relief pain syndrome. Human J Trad Chin Med, 2013, 29(5): 148-149.

17 Gao YF. The effects of cognitive behavior with music in relieving the native emotion and quality of sleeping in patients after the surgery of intracranial tumors. Modern J Integr Trad Chin Western Med, 2016, 25(18): 2047-2049.

18 Cao YH, Hou LL, Li YM, et al. The effects of Shang tune of music in improving insomnia in patients with lung cancer during chemotherapy. Shanghai Nurs, 2015, 15(6): 26-28.

19 Di JY. The application of Aromatherapy with music for colorectal cancer. J Integr Trad Chin Western Med, 2017, 3(4): 19-21.

20 Koelsch S. Towards a neural basis of music -evoked emotions. Trends Cogn Sci, 2010, 14(3): 131-137.

21 Shi HF, Luo GQ, Li L. Clinical research progress of acupuncture therapy for pain syndrome, Hunan traditional Chinese medicine news 2013; 29(5): 148149.

22 Chinese Psychiatric Association. Classification and Diagnostic Criteria for Mental Disorders in China. Nanjing: Southeast University Press, 1995: 94. 